\title{
A high-resolution lithospheric magnetic field model over southern Africa based on a joint inversion of CHAMP, Swarm, WDMAM, and ground magnetic field data
}

\author{
Foteini Vervelidou $^{1}$, Erwan Thébault ${ }^{2}$, and Monika Korte ${ }^{1}$ \\ ${ }^{1}$ Helmholtz Centre Potsdam-GFZ German Research Centre for Geosciences, Section 2.3 Geomagnetism, Telegrafenberg, \\ 14473 Potsdam, Germany \\ ${ }^{2}$ Université de Nantes, Laboratoire de Planetologie et Geodynamique, 44322 Nantes Cedex 3, France
}

Correspondence: Foteini Vervelidou (foteini@gfz-potsdam.de)

Received: 16 March 2018 - Discussion started: 5 April 2018

Revised: 19 June 2018 - Accepted: 26 June 2018 - Published: 16 July 2018

\begin{abstract}
We derive a lithospheric magnetic field model up to equivalent spherical harmonic degree 1000 over southern Africa. We rely on a joint inversion of satellite, near-surface, and ground magnetic field data. The input data set consists of magnetic field vector measurements from the CHAMP satellite, across-track magnetic field differences from the Swarm mission, the World Digital Magnetic Anomaly Map, and magnetic field measurements from repeat stations and three local INTERMAGNET observatories. For the inversion scheme, we use the revised spherical cap harmonic analysis (R-SCHA), a regional analysis technique able to deal with magnetic field measurements obtained at different altitudes. The model is carefully assessed and displayed at different altitudes and its spectral content is compared to high-resolution global lithospheric field models. By comparing the shape of its spectrum to a statistical power spectrum of Earth's lithospheric magnetic field, we infer the mean magnetic thickness and the mean magnetization over southern Africa.
\end{abstract}

\section{Introduction}

The increasing availability of magnetic field measurements, both at satellite altitude and near or at the Earth's surface, makes it possible to improve models of the lithospheric magnetic field periodically, both in terms of accuracy and spatial resolution. Marine and aeromagnetic measurements, because of their proximity to lithospheric sources, are capable of capturing the small-scale features of the lithospheric magnetic field well. However, its large-scale contributions are not accessible by means of these measurements because of their limited spatial extent. Low Earth orbiting magnetic satellite missions, such as CHAMP (Reigber et al., 2002) and the ongoing multi-satellite mission Swarm (Friis-Christensen et al., 2006; Olsen et al., 2013), complement near-surface measurements by offering a consistent global view of the large-scale features of the lithospheric magnetic field. The MF7 model, for example, based on CHAMP satellite measurements is derived up to spherical harmonic ( $\mathrm{SH}$ ) degree 133 (Maus et al., 2008), and the LCS-1 model based on a joint consideration of CHAMP along-track differences and Swarm along- and across-track differences pushes this limit to SH degree 185 , although with a significant loss of energy beyond SH degree 133 (Olsen et al., 2017).

Satellite measurements and a coordinated international effort to collect and merge all publicly available near-surface measurements led to the first global grid of lithospheric field anomalies, the World Digital Magnetic Anomaly Map (WDMAM), which was published in 2007 (Korhonen et al., 2007) by the WDMAM Task Force under the auspices of the International Association of Geomagnetism and Aeronomy (IAGA) and the Commission for the Geological Map of the World (CGMW). The MF5 model (Maus et al., 2007) based on CHAMP measurements was used to account for its long wavelengths. Based on a different way of processing and merging the marine and aeromagnetic measurements, Maus et al. (2009) derived another anomaly grid, the EMAG2, which was converted by Maus (2010) to an SH model up to degree 720 (see https://www.ngdc.noaa.gov/geomag/EMM/ 
for an updated version, the EMM2017, released on 5 July 2017, last access: 12 July 2018). In 2015, the second version of the WDMAM was published (Lesur et al., 2016; http://www.wdmam.org/, last access: 12 July 2018). New data sets were added and the data gaps over the oceans were handled in a different way (see Dyment et al., 2015). The second version of the WDMAM has been converted to an SH model up to degree 800 (henceforth termed WDMAMv2SH800).

These global models represent the first attempt to merge the information content of satellite and near-surface measurements in the form of SH models. However, this merging is not carried out by a joint inversion of satellite and near-surface measurements, mainly because of the existence of wide data gaps that lead to numerical instabilities. The high inhomogeneity in data availability calls for regional modeling approaches that are flexible in adapting their spatial resolution to the available data coverage. Over regions well covered by magnetic field measurements, regional models can achieve higher accuracy and spatial resolution than global models. A review of available regional modeling techniques in the framework of geomagnetism is given by Schott and Thébault (2011). More recent regional techniques to jointly model data at different altitudes include the vector Slepian functions (Plattner and Simons, 2014; Plattner and Simons, 2017) and the use of equivalent potential field sources (Kother et al., 2015; Kother, 2017). Once lithospheric field models are derived, they can be analyzed in the spatial and spectral domains in order to infer the properties of the underlying sources, like Curie depth and magnetization (e.g., Whaler, 2003; Ravat et al., 2007; Bouligand et al., 2009; Rajaram et al., 2009; Scheiber-Enslin et al., 2014, Vervelidou and Thébault, 2015).

In this study, we focus on the southern part of Africa, which is well covered by near-surface measurements, and we opt for the revised spherical cap harmonic analysis (RSCHA, Thébault et al., 2006) for modeling the lithospheric magnetic field. We use our model to infer the thickness of the magnetized layer, henceforth termed the magnetic thickness, and the magnetization intensity. In Sect. 2 of this paper we present the input data set. In Sect. 3, we describe the modeling method. Then, we present the model with plots at various altitudes and assess it by showing its power spectrum, the residuals for each data set, and the statistics of the inversion. Further on, its power spectrum is used as a means for inferring the mean magnetic thickness and mean magnetization of the region under study. Finally, we discuss our findings and present conclusions and perspectives from our study.

\section{Data}

The combined use of magnetic field measurements taken at different altitudes requires careful data processing in order to maximize their compatibility (see, e.g., Ravat et al., 2002). In the following sections we describe the dedicated processing scheme of each data type included in our joint inversion. All data are selected within a spherical cap of $\theta_{0}=15^{\circ}$ centered at longitude $22.5^{\circ} \mathrm{E}$ and latitude $25^{\circ} \mathrm{S}$.

\subsection{Satellite data}

We consider magnetic field CHAMP satellite measurements that were selected and processed following the procedure described by Lesur et al. (2010). The data were rotated into the solar magnetic (SM) Cartesian coordinate system (in this coordinate system the $Z$ SM axis coincides with the geomagnetic dipole axis and points to the north, the Sun-Earth line lies on the $x-z$ plane, and the $Y$ SM axis is perpendicular to the Earth-Sun line and points towards dusk; see Laundal and Richmond, 2017); only the $X$ and $Y$ SM components were kept. This was done in order to avoid contamination by the ring current (see Lesur et al., 2008). In addition, data were selected only for local times between 23:00 and 05:00, when the $Z$ component of the interplanetary magnetic field (IMF) was positive, the norm of the vector magnetic disturbance index (VMD; Thomson and Lesur, 2007) less than $20 \mathrm{nT}$, and the norm of its derivative less than $100 \mathrm{nT} \mathrm{day}^{-1}$. From the selected data, the GRIMM lithospheric field model (Lesur et al., 2013) from SH degree 17 to $\mathrm{SH}$ degree 80 was subtracted. This was done in order to avoid a spectral leakage of the lithospheric field into the secular variation model (see, e.g., Lesur et al., 2010). The residuals were used to construct a time-dependent core field model up to SH degree 18 (with splines of order 6 with a knot spacing of 6 months as the temporal basis function), a static lithospheric field model up to SH degree 30, an external static field model up to SH degree 20 , and a time-varying external field model based on three different parameterizations. A slowly varying external field component was considered by solving for an axial dipole external term in the GSM coordinate system every 10 years. The more rapidly varying external field components were considered by solving for the SH degree 1 coefficients in the SM coordinates every 100 days. The fact that these bins were 100 days large reduced the risk of leakage due to a correction of a track-by-track type (see Thébault et al., 2012). Finally, the even more rapidly varying external fields were accounted for by solving for the scaling coefficients of the SVMD index, the satellite-based version of the VMD index. This index is obtained by calculating the mean value of the measured magnetic field over each orbit and by subsequently normalizing these values in bins of 100 days (see Kunagu et al., 2013). To the vector residuals of this modeling procedure the initially removed lithospheric field model was added back. The final CHAMP data set comprises 13229 triplets of vector data varying between 266 and $475 \mathrm{~km}$ of altitude.

The selection and correction procedure of Swarm magnetic field measurements closely follows the one described in Thébault et al. (2016) for middle and low latitudes. Magnetic field measurements taken by the lowest pair of Swarm 
satellites (Alpha and Charlie) are considered between March 2014 and December 2015. The diurnal ionospheric field contribution is minimized by keeping nighttime data only (local time between 23:00 and 05:00 and Sun at least $10^{\circ}$ below the horizon). Data taken during disturbed magnetic conditions are rejected by selecting only times for which the Dst index is lower than $5 \mathrm{nT}$, with a time variation smaller than $5 \mathrm{nT}$ over the three previous hours, and for which the Kp index is lower than 2 . The selected measurements are then corrected for independent main (Rother et al., 2013) and magnetospheric field models (Hamilton, 2013) and are further corrected on a track-by-track basis. The residuals, vector and scalar, are used to construct finite differences between the measurements taken by Swarm Alpha and Charlie (hereafter called "across-track differences"). These differences simulate the east-west gradient, which is very efficient in filtering out the remaining rapid variations of the external field (Kotsiaros and Olsen, 2012). The final Swarm data set contains 27699 triplets of vector Swarm across-track differences and 27690 scalar Swarm across-track differences varying between 460 and $480 \mathrm{~km}$ of altitude.

\subsection{Aeromagnetic data}

Since 1970, coordinated efforts have been made by African countries and collaborators towards the aeromagnetic mapping of southern Africa. This African magnetic mapping project (Barritt, 1993) eventually led to the SaNaBoZi aeromagnetic grid; the name stands for South Africa, Namibia, Botswana, and Zimbabwe (Stettler et al., 2000). The SaNaBoZi grid and more recently acquired grids over Zambia and Mozambique (see Webb, 2009) as well as marine data offshore of South Africa have been compiled and are part of the second version of the WDMAM (Dyment et al., 2015; Lesur et al., 2016) used in this study. In order to reduce the data volume and balance the number of near-surface and satellite measurements, the respective grid is resampled to $0.1^{\circ}$, leading to 77626 aeromagnetic and marine scalar measurements.

\subsection{Magnetic observatory and repeat station data}

Our ground data set comprises magnetic measurements from southern African repeat stations and geomagnetic observatories as processed by Korte and Mandea (2016). From 2005 to 2015 annual repeat station surveys were carried out in the framework of a collaboration between Hermanus Magnetic Observatory (now SANSA Space Science) and the German Research Center for Geosciences, GFZ. The surveys comprised 40 stations in South Africa, Namibia, and Botswana. Using an on-site variometer at all stations, the measurements were reduced to (quiet) nighttime averages (see details in Korte et al., 2007). Here, we consider the data up to 2009 and use version 3 of the GRIMM series (Lesur et al., 2010; Mandea et al., 2012) to eliminate core (up to SH degree 14) and

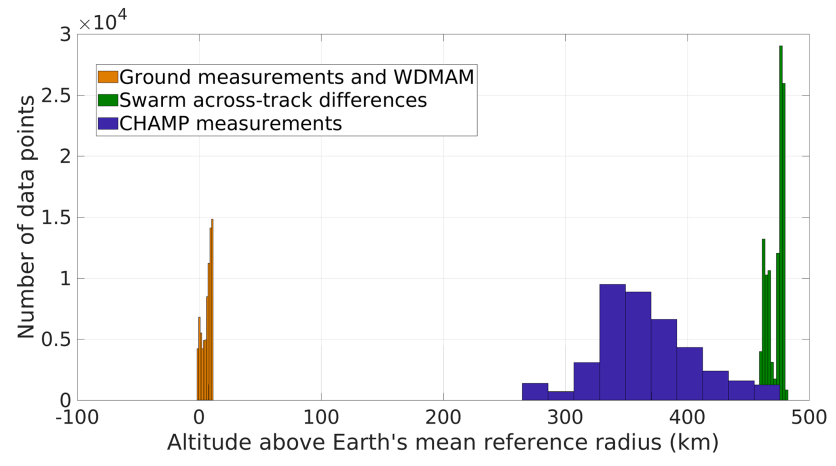

Figure 1. Histograms of the different types of magnetic field data included in our model as a function of their altitude above the Earth's mean reference radius (according to the geocentric reference frame).

magnetospheric field contributions from the data. The final data set comprises up to five vector magnetic anomaly estimates for each location, in some cases fewer due to gaps in the annual series or the rejection of obvious outliers. There are four INTERMAGNET observatories in the southern African region, but as the first available annual mean value from observatory Keetmanshoop in Namibia dates to 2009.5, measurements from this observatory were not included in the data set. For each one of the other three magnetic observatories, which are Hermanus and Hartebeesthoek in South Africa and Tsumeb in Namibia, nine vector lithospheric anomaly estimates were obtained by subtracting the GRIMM3 core and magnetospheric predictions from the annual mean values. In total, 161 triplets of ground vector measurements over 37 repeat stations and 27 triplets of annual mean values from 3 INTERMAGNET observatories were included in the ground data set.

The input data set is summarized in Table 1. Additionally, Fig. 1 shows a histogram of the different data types as a function of altitude above the Earth's mean reference radius.

\section{Method}

Revised spherical cap harmonic analysis (Thébault et al., 2006) is used for modeling the lithospheric magnetic field inside the volume of a spherical cone. The cone is defined as the intersection of two spherical caps defined by two concentric spheres of different radii and an infinite cone of halfangle aperture $\theta_{0}$, whose apex lies at the Earth's center. In contrast to spherical cap analysis (Haines, 1985) that solves one boundary problem on the lateral boundaries of a spherical cap, the R-SCHA technique solves two boundary problems: one on the lateral boundaries of the spherical cone and one on its upper and lower boundaries. Each one of these two boundary problems generates one set of basis functions, the Legendre and the Mehler basis functions, respectively. The Legendre basis functions are laterally oscillating functions, 
Table 1. The amount of input data per data type.

\begin{tabular}{|c|c|c|c|c|}
\hline & $\begin{array}{r}\text { Vector } \\
\text { (number of triplets) }\end{array}$ & Scalar & $\begin{array}{r}\text { Vector across-track differences } \\
\text { (number of triplets) }\end{array}$ & Scalar across-track differences \\
\hline Satellite CHAMP & 13229 & & & \\
\hline Satellite mission Swarm & & & 27699 & 27690 \\
\hline WDMAM & & 77626 & & \\
\hline Magnetic observatories & 27 & & & \\
\hline Repeat stations & 161 & & & \\
\hline
\end{tabular}

while the Mehler basis functions are radially oscillating and can account for the upward-downward continuation operation of the potential field inside the cone. The magnetic field $\boldsymbol{B}=\boldsymbol{B}_{1}+\boldsymbol{B}_{2}$ is the sum of the magnetic field projected on these two basis functions, where $\boldsymbol{B}_{1}$ is written as the gradient of a series of the Legendre basis functions:

$$
\begin{gathered}
\boldsymbol{B}_{1}(r, \theta, \phi)=-\nabla\left\{\alpha \sum _ { k \geq 1 } \sum _ { k \leq m \leq k } \left[\left(\frac{\alpha}{r}\right)^{n_{k}+1} G_{n_{k}}^{i, m}\right.\right. \\
\left.\left.+\left(\frac{r}{\alpha}\right)^{n_{k}} G_{n_{k}}^{e, m}\right] \beta_{n_{k}}^{m}(\theta, \phi)\right\},
\end{gathered}
$$

with

$\beta_{n_{k}}^{m}(\theta, \phi)= \begin{cases}\cos m \phi P_{n_{k}}^{m}(\theta), & 0 \leq m \leq k \\ \sin |m| \phi P_{n_{k}}^{m}(\theta), & -k \leq m \leq-1,\end{cases}$

where $P_{n_{k}}^{m}(\theta)$ represents the generalized Legendre functions of integer order $m$ and real degree $n_{k}, G_{n_{k}}^{i, m}$ and $G_{n_{k}}^{e, m}$ the harmonic coefficients of the corresponding order and degree to be estimated via the inverse problem, and $\boldsymbol{B}_{2}$ is written as the gradient of a series of the Mehler basis functions:

$\boldsymbol{B}_{2}(r, \theta, \phi)=-\nabla\left\{\alpha \sum_{p \geq 0} \sum_{p \leq m \leq p} R_{p}(r) G_{p}^{m} \gamma_{p}^{m}(\theta, \phi)\right\}$,

with

$R_{p}(r)=$

$\left\{\sqrt{\frac{\alpha}{r}\left[\frac{2 \pi p}{\log \left(\frac{\beta}{\alpha}\right)} \cos \left(p \pi \frac{\log \left(\frac{r}{\alpha}\right)}{\log \left(\frac{\beta}{\alpha}\right)}\right)+\sin \left(p \pi \frac{\log \left(\frac{r}{\alpha}\right)}{\operatorname{const},}\right)\right],} \quad \begin{array}{l}p \geq 1 \\ \log \left(\frac{\beta}{\alpha}\right)\end{array}\right.$

and

$\gamma_{p}^{m}(\theta, \phi)=\left\{\begin{array}{l}\cos m \phi K_{p}^{m}(\theta), 0 \leq m \leq p \\ \sin |m| \phi K_{p}^{m}(\theta),-p \leq m \leq-1,\end{array}\right.$

where $K_{p}^{m}(\theta)$ represents the conical or Mehler functions of integer order $m$ and degree $n_{p}, \alpha$ and $\beta$ are the radii of the lower and upper spherical caps, respectively, and $G_{p}^{m}$ represents the harmonic coefficients of the corresponding order and degree to also be estimated through the inverse problem.
This problem can be written as a linear system of equations:

$\mathbf{G m}=\boldsymbol{d}$,

where $\boldsymbol{d}$ is the vector of the data, $\boldsymbol{m}$ is the set of coefficients to be determined, and $\mathbf{G}$ is the matrix of the basis functions. The scalar part of the input data set is linearized through a projection on the ambient field (see, e.g., Blakely, 1996), here taken to be the GRIMM2 core field model centered on the 2005.5 epoch (Lesur et al., 2010).

The data are inverted in an iteratively re-weighted least square sense with Huber weighting (see, e.g., Farquharson and Oldenburg, 1998; Sabaka et al., 2004), and no regularization is applied. In this case, the coefficients $\boldsymbol{m}$ are given by

$\boldsymbol{m}=\left(\mathbf{G}^{T} \mathbf{W} \mathbf{G}\right)^{-1} \mathbf{G}^{T} \mathbf{W} \boldsymbol{d}$,

where $\mathbf{W}$ is the weighting matrix, which changes at every iteration. It is diagonal and its element $i$ at iteration $j$ is given by

$w_{i j}=\frac{1}{\sigma_{i}^{2}} \min \left(\frac{c \sigma_{i}}{\left\|e_{i j}\right\|}, 1\right)$,

where $\sigma_{i}$ is the standard deviation of the $i$ th point, $e_{i j}$ the residual of the $i$ th point at iteration $j$, and $c$ a constant here set to 1.5 (following Sabaka et al., 2015). Taking into account the expected accuracy, number of points, and typical values due to distance from the sources of each data type, the standard deviation for the ground data is taken to be equal to $20 \mathrm{nT}$ for all three components, for the aeromagnetic data equal to $40 \mathrm{nT}$, and for the satellite data equal to $2 \mathrm{nT}$ for all three components. The truncation index for $k$ and $m$ in Eq. (1) is set to 80 , which allows for the description of the field up to a $40 \mathrm{~km}$ horizontal wavelength. The truncation index for $p$ and $m$ in Eq. (3) is set to 9. This number is constrained by the availability of data at different altitudes (see paragraph 5.2.2 of Vervelidou (2013), for more details). The radius of the lower and upper spherical cap was set to -10 and $500 \mathrm{~km}$, respectively, in order to avoid artifacts due to edge effects. 
In order to estimate the spectral content of the regional model, we compute its R-SCHA surface power spectrum according to Vervelidou and Thébault (2015), their Eq. (10):

$$
\begin{aligned}
& \frac{E_{\vartheta \Omega_{\rho}}}{S_{\vartheta \Omega_{\rho}}}=\sum_{m=0}^{\infty} \frac{\left(1+\delta_{m, 0}\right)}{2\left(1-\cos \theta_{0}\right)} \sum_{k=0}^{\infty} \\
& \quad\left\{\left(n_{k}+1\right)\left(2 n_{k}+1\right)\left(\frac{a}{\rho}\right)^{2 n_{k}+4}\left[\left(G_{n_{k}}^{i, m}\right)^{2}+\left(H_{n_{k}}^{i, m}\right)^{2}\right]\right. \\
& \left.\quad+n_{k}\left(2 n_{k}+1\right)\left(\frac{\rho}{a}\right)^{2 n_{k}-2}\left[\left(G_{n_{k}}^{e, m}\right)^{2}+\left(H_{n_{k}}^{e, m}\right)^{2}\right]\right\}\left\|P_{n_{k}}^{m}\right\|^{2},
\end{aligned}
$$

where $\rho$ is the radius of the sphere on the surface of which we calculate the power spectrum, $\vartheta \Omega_{\rho}$ is that surface, and $S_{\vartheta \Omega_{\rho}}$ its area.

This power spectrum is equivalent to an $\mathrm{SH}$ power spectrum (Lowes, 1966), with the only difference that the degrees $n_{k}$ are real numbers and depend on the order $m$. Therefore, each term of the double sum in Eq. (9) corresponds to a different degree $n_{k}$. In order to be able to associate the terms of this power spectrum to a specific wavelength, we bin together the harmonic coefficients corresponding to degrees with close numerical values. Each bin is taken to be $\frac{\pi}{\theta_{0}}$ large, following Vervelidou and Thébault (2015), and its characteristic wavelength is related to its mean degree $\bar{n}$ according to the known approximation (see Backus et al., 1996):

$\bar{\lambda} \approx \frac{2 \pi \rho}{\bar{n}+\frac{1}{2}}$.

\section{Results}

\subsection{Statistics of the multiscale inversion}

The residuals of each data type after the inversion are shown in Figs. 2 and 3. Figure $2 \mathrm{a}$ and $\mathrm{b}$ show the residuals of the CHAMP and Swarm data sets, respectively. While in the CHAMP data set all three components show satellite tracks being partially or entirely contaminated by external field noise, this is not the case for the residuals of the Swarm differences, especially for the $Z$ component.

The residuals between the model and the WDMAM, shown in Fig. 2d, are of short wavelengths and reflect the difference between the spatial resolution of our model and the spatial resolution of the input grid. Residuals over the oceanic region are likely due to large gaps in marine magnetic field measurements. In the second version of the WDMAM used here, these gaps have been filled in by means of the magnetization model of Dyment et al. (2015), which is based on considerations concerning the age of the ocean floor, tectonic motions, and geomagnetic polarity reversals.

Figure 3 shows the predictions of our model (red circles) and the mean of the measurements (blue squares) at each repeat station and magnetic observatory considered in our inversion for the $X, Y$, and $Z$ component. The abbreviations used here are those used by Korte and Mandea (2016) (see their Table 3). According to this figure, our model is in close agreement with the actual measurements. This is a mutual confirmation of both the accuracy of our model and the measurements. The spatial distribution of the residuals is shown in Fig. 2c. According to this figure, the residuals do not exhibit any specific spatial pattern.

The root mean square deviation (RMSD) for each data type and each component is summarized in Table 2 . We see that the RMS of all three components of the ground data is below the initial standard deviation, set to $20 \mathrm{nT}$. This shows that the location of the repeat stations do not lie on very localized anomalies, which is actually one of the objectives when selecting repeat station locations. The RMS of the $Z$ component is the smallest, which is expected as the horizontal components $X$ and $Y$ are usually more contaminated by external field noise. The RMS of the WDMAM is larger than the initial standard deviation, set to $40 \mathrm{nT}$. This is because the WDMAM grid has features with a spatial scale smaller than our model is able to depict. All three components of the CHAMP data are resolved within the initial standard deviation. The Swarm differences show, as expected, smaller misfit values than the CHAMP measurements. The second row of Table 2 shows the correlation coefficient between the model predictions and the data separately for each data type and component. We see that all data types are well resolved, with correlation coefficients above 0.7 . This indicates a good level of compatibility between all different data sets. The lowest correlation is found for the $X$ and $Y$ components of the CHAMP data and the $Y$ component of the Swarm across-track differences (between 0.7 and 0.77), which is expected due to the external field contamination of the horizontal components in satellite measurements also visible in Fig. 2.

\subsection{The model in the spatial and spectral domain}

Figure 4 shows the model prediction for $X, Y$, and $Z$ components at the Earth's mean reference radius. Figure 5 shows the model prediction for the $Z$ component at various altitudes above the Earth's mean reference radius. The results over Angola and adjacent regions up to $50 \mathrm{~km}$ of altitude are shaded with grey because WDMAM in that region does not include near-surface data and is filled in only with the values of the satellite-based model GRIMM_L120 (Lesur et al., 2013, 2016). Such a contrast in the spectral content of the available magnetic field measurements introduces artificial small-scale oscillations, an effect known as ringing. In order to avoid a possible overinterpretation of these structures we exclude them from our model. The different components highlight structures of different orientation and the maps at different altitudes carry information about the lateral and vertical extent of the sources. These maps are discussed in more detail in the Discussion section.

Figure 6 shows the R-SCHA power spectrum of the model (blue line) up to equivalent SH degree 1000 (spatial 
(a) CHAMP
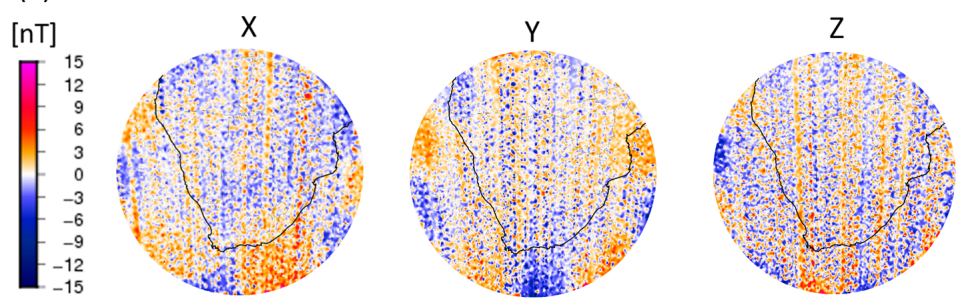

(b) Swarm across-track differences
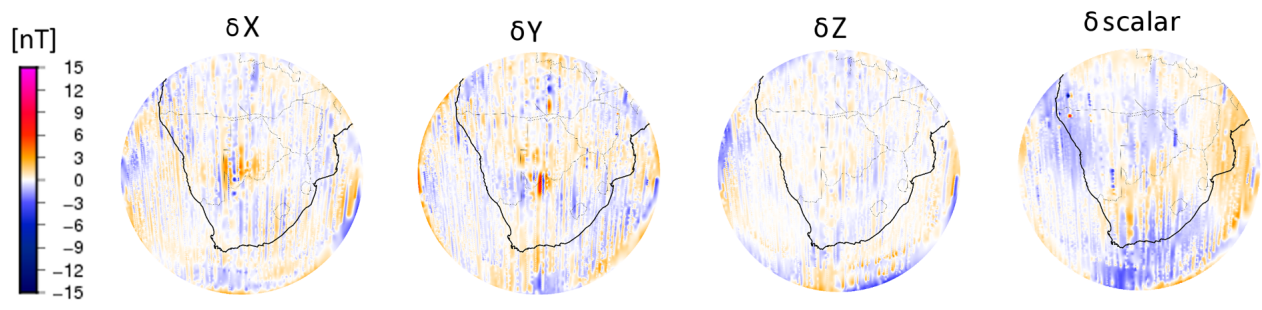

(c) Repeat station and observatory data
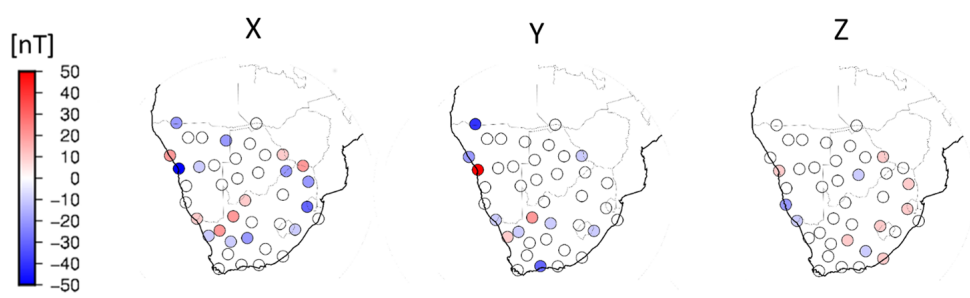

(d) WDMAM

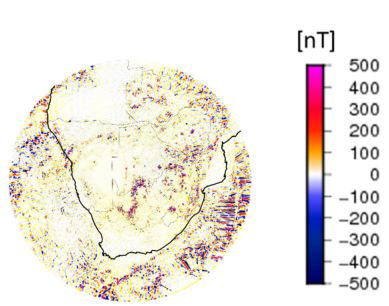

Figure 2. Residuals between our model and the input data for (a) the CHAMP vector measurements, (b) the Swarm across-track differences of the vector and scalar measurements, (c) the repeat station and observatory vector measurements, and (d) the WDMAM grid points. Note the different color scales.

Table 2. The root mean square deviation (RMSD) and the correlation coefficient between our model and the input data.

\begin{tabular}{|c|c|c|c|c|c|c|c|c|c|c|c|}
\hline & \multicolumn{3}{|c|}{ Ground data } & \multirow[t]{2}{*}{ WDMAM } & \multicolumn{3}{|c|}{ CHAMP } & \multicolumn{4}{|c|}{ Swarm across-track differences } \\
\hline & $X$ & $Y$ & $Z$ & & $X$ & Y & $Z$ & $X$ & $Y$ & $Z$ & Scalar \\
\hline RMSD (nT) & 13.6 & 12.7 & 6.8 & 67.7 & 2.3 & 2.2 & 2.3 & 0.6 & 0.6 & 0.4 & 0.8 \\
\hline Correlation coefficients & 0.99 & 0.99 & 1 & 0.8 & 0.7 & 0.77 & 0.84 & 0.85 & 0.73 & 0.95 & 0.83 \\
\hline
\end{tabular}

wavelength $40 \mathrm{~km})$. It is compared to the power spectra of WDMAMv2-SH800 (Lesur et al., 2016) and the EMM2017 (see https://www.ngdc.noaa.gov/geomag/EMM/, last access: 12 July 2018) given in red and green, respectively. The regional R-SCHA power spectra over southern Africa of these two global models were calculated from their vector predictions at the Earth's mean reference radius within the same spherical cap and for the same inversion parameters used for the derivation of our regional model. Given the choice of $k_{\max }=80$, the comparison is only valid up to degree $\bar{n}=340$, beyond which the spectral content of global models is not fully recovered (for details see Vervelidou, 2013, chap. 4). To denote the transition limit, we use solid lines for the spectra up to $\bar{n}=340$ and dashed lines beyond this degree. According to this figure, the power spectrum of the regional model up to $\mathrm{SH}$ degree 100 , which is the bandwidth constrained by satellite data, lies between the power spectra of WDMAMv2-SH800 and EMM2017. Starting from SH degree 100 , the regional model is more energetic than the two global models. As expected, the global models carry no energy beyond degree 800 , while the regional model carries energy over a larger bandwidth.

\subsection{Magnetic thickness and magnetization estimates}

We use the power spectrum of our model to estimate the mean magnetic thickness and the mean magnetization over southern Africa. Traditionally, such studies rely on Fourier power spectra (see, e.g., Ravat et al., 2007, and references therein; Rajaram et al., 2009, Bansal et al., 2011, Salem et al., 2014). Fourier spectra rely on the flat Earth approximation and are therefore valid only on local scales. The R- 

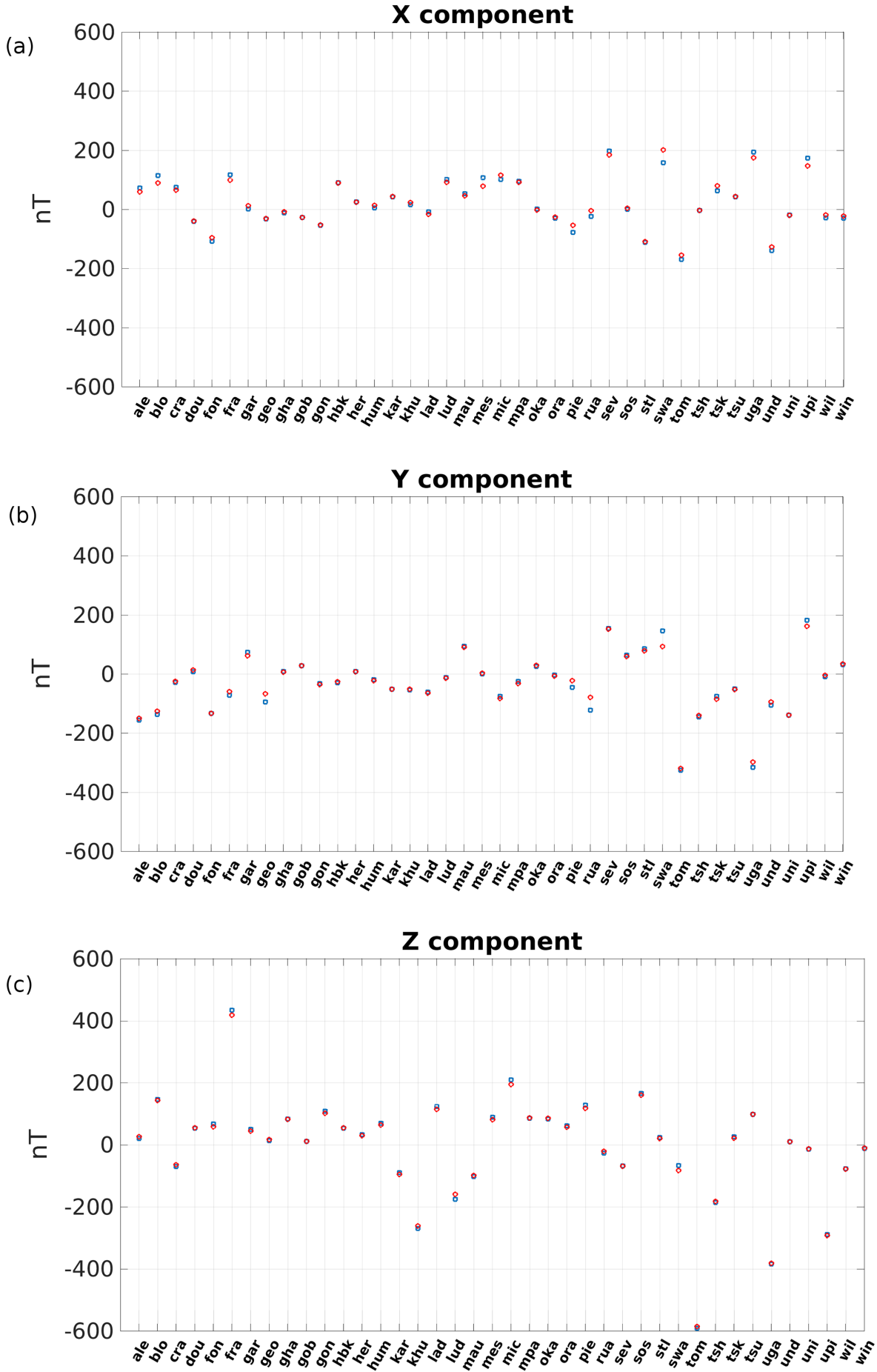

Figure 3. The predictions of our model over the ground measurement points (red circles) and the mean of the measurements (blue squares) for the $X, Y$, and $Z$ components. The abbreviations follow Table 3 by Korte and Mandea (2016). 

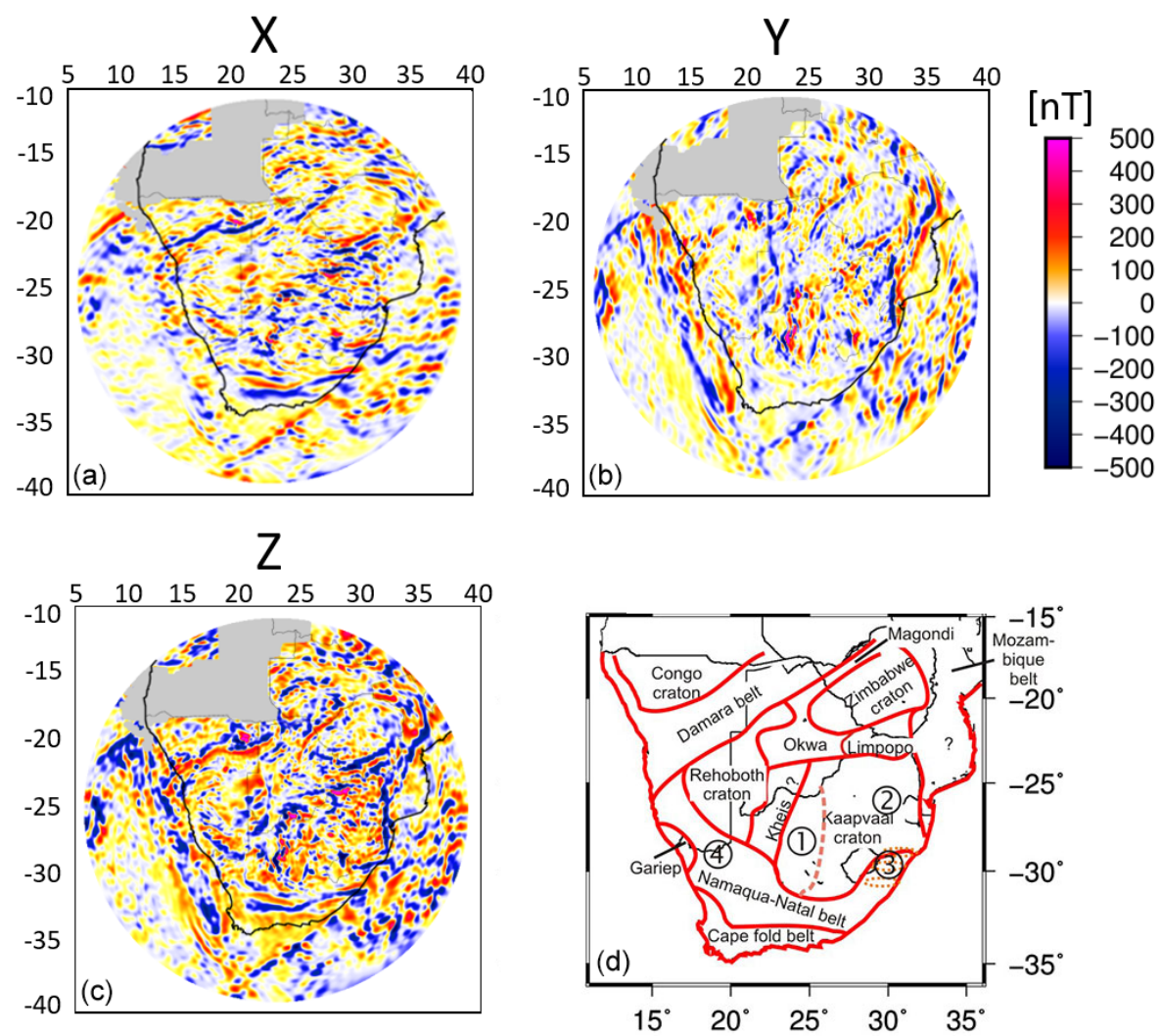

Figure 4. (a-c) The $X, Y$, and $Z$ component of our lithospheric field model plotted at the Earth's mean reference radius. The part shaded with grey corresponds to a region over which WDMAM is void of near-surface data. (d) Outline of large-scale tectonic features; from Fig. 8 by Korte and Mandea (2016).

SCHA power spectrum is defined in spherical coordinates and therefore offers a more accurate description of the energy per wavelength over regional scales. To infer the mean magnetic thickness and magnetization estimates, we fit the observational power spectrum to the statistical power spectrum of the Earth's lithospheric magnetic field derived by Thébault and Vervelidou (2015). This statistical expression is based on the following three assumptions: the thickness of the magnetized shell is finite and constant, the inducing field is axial dipolar, and the power spectrum of the susceptibility follows a power law. This expression has been shown to fit high-resolution lithospheric field models well (see Vervelidou, 2013, Sect. 4.3; Thébault and Vervelidou, 2015, their Fig. 4; Vervelidou and Thébault, 2015, their Fig. 5).

The misfit functional between the observational and the statistical power spectra is defined as follows (Voorhies et al., 2002, Eq. 14a)

$\chi^{2}=\sum_{n}\left[\ln \left(R_{n}\right)-\ln \left(E\left\{R_{n}\right\}\right)\right]^{2}$,

where $R_{n}$ is the observational power spectrum and $E\left\{R_{n}\right\}$ its expected value according to the statistical expression of Thébault and Vervelidou (2015) (their Eq. 26), which depends on three parameters: the thickness of the magnetized layer, the magnetization intensity, and the power law that characterizes the power spectrum of the susceptibility distribution. The co-estimation of the three unknown parameters is unstable (see, e.g., Bouligand et al., 2009). A possible way to alleviate some of the ambiguity is to rely on a priori information about the susceptibility power law. Here, we set its value to 1.36, in agreement with the global estimate of Thébault and Vervelidou (2015) based on the susceptibility map of Hemant and Maus (2005) and the NGDC-720 lithospheric field model (Maus, 2010).

We calculate the misfit functional $\chi^{2}$ for a variety of different thickness and magnetization values. This provides us with the 2-D function shown in Fig. 7a. As expected, there is a trade-off between the magnetization and the magnetic thickness. We find that Eq. (11) has its minimum for an equivalent magnetization of $0.7 \mathrm{~A} \mathrm{~m}^{-1}$, which is twice as large as the global average estimated by Thébault and Vervelidou (2015), and for a magnetic thickness equal to $16 \mathrm{~km}$. Considering the misfit values up to 3 standard deviations above the misfit minimum, we find that the mean magnetic thickness lies between 11 and $22 \mathrm{~km}$ and the mean magnetization between 0.6 and $0.9 \mathrm{~A} \mathrm{~m}^{-1}$ (the respective values are marked with black in Fig. 7a). This estimate is in close agreement with the $20 \mathrm{~km}$ mean magnetic 

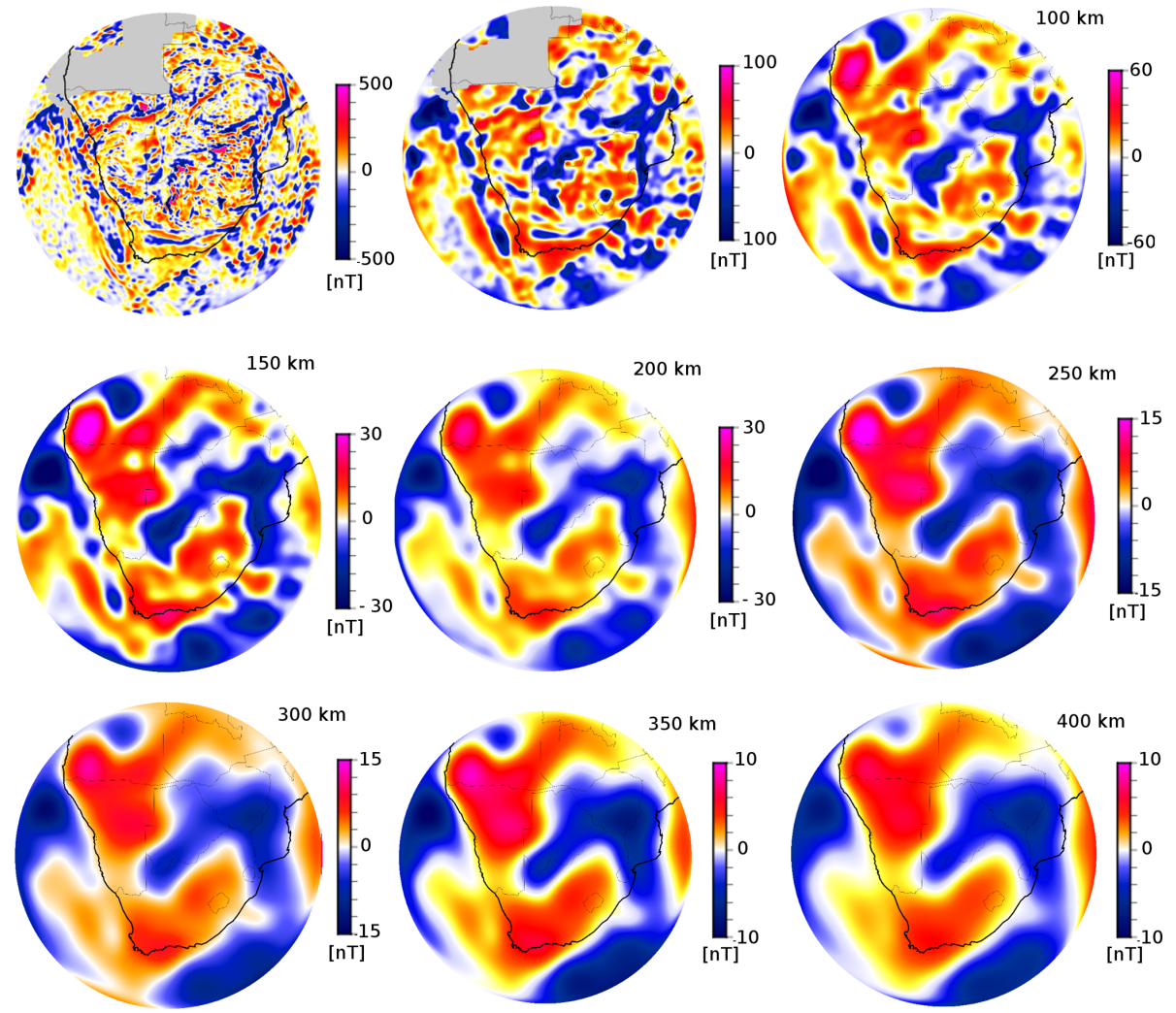

Figure 5. The $Z$ component of our lithospheric field model plotted at different altitudes above the Earth's mean reference radius. The part shaded with grey corresponds to a region over which WDMAM is void of near-surface data. Note the different color scales.

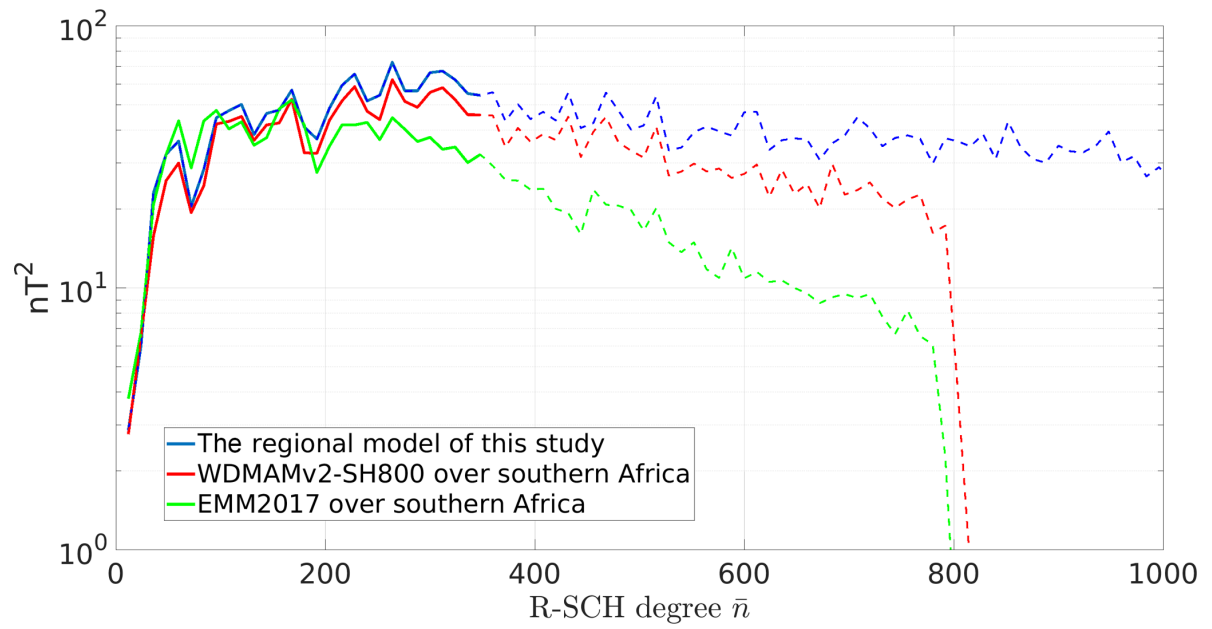

Figure 6. The power spectrum of our regional lithospheric field model (blue curve) and the power spectra of the global WDMAMv2-SH800 and EMM2017 calculated over the region of interest (red and green curves, respectively). The solid line is used for the bandwidth over which the comparison between the spectra of the regional and global models is valid (see text for details).

thickness proposed by Maus et al. (1997) following a similar spectral analysis but based on a Cartesian statistical expression for the power spectrum of the Earth's lithospheric magnetic field and a Fourier observational power spectrum over southern Africa given by Whaler (1994).

\section{Discussion}

The regional field model derived in this study offers insights into several aspects of lithospheric field modeling. The first concerns the contamination of lithospheric field models by 

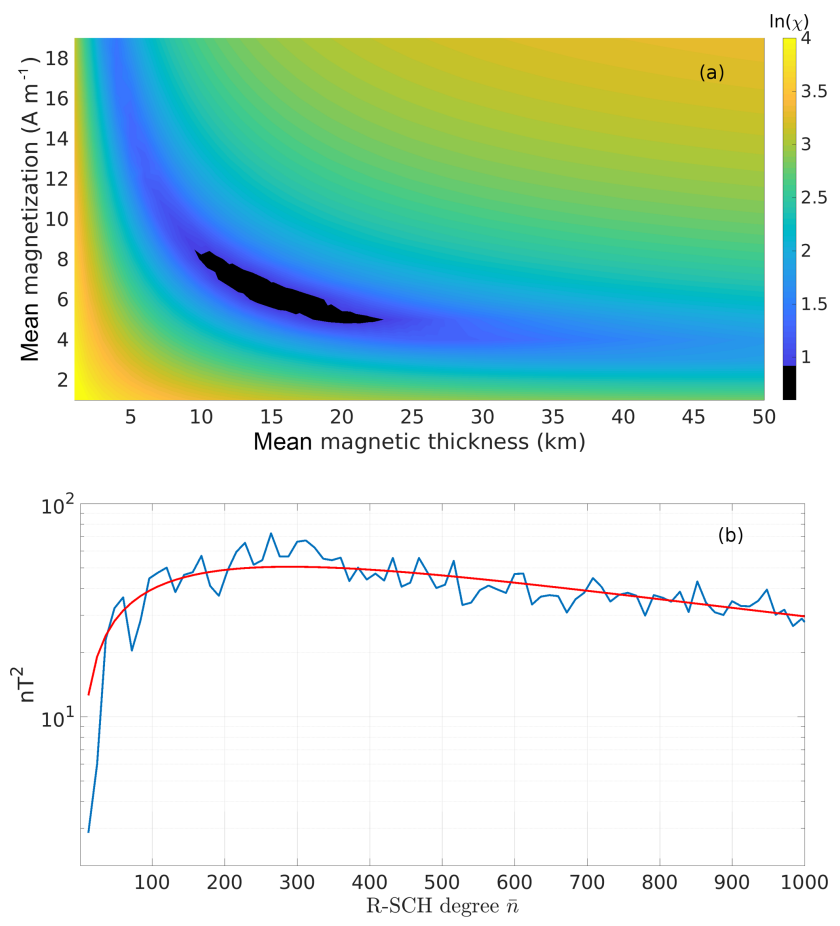

Figure 7. (a) The natural logarithm of the misfit (Eq. 11) between the R-SCHA power spectrum of our model and the statistical expression of Thébault and Vervelidou (2015) for a range of values for the mean magnetic thickness and magnetization. Values within the $3 \sigma$ confidence interval are marked with black. (b) The R-SCHA power spectrum of our model (blue curve) and the statistical expression for $16 \mathrm{~km}$ magnetic thickness and $0.7 \mathrm{~A} \mathrm{~m}^{-1}$ magnetization (red curve).

external field sources (see Finlay et al., 2017, and Thébault et al., 2017, for reviews on this topic). One major source of contamination is the magnetic field generated by the magnetospheric ring current. The strength of this current fluctuates rapidly under the effect of the solar wind, even during magnetically quiet times, and so does the associated magnetic field. A single satellite cannot distinguish between these time variations and the small spatial-scale variations of the lithospheric field because of its constantly moving position. As this signal of magnetospheric origin changes from one track to another (due to this signal's temporal variation), it introduces offsets between adjacent satellite tracks. This effect is illustrated by the residuals between the model and the satellite CHAMP measurements shown in Fig. 2a. Figure 2b, depicting the residuals between the model and the Swarm measurements, does not show similar prominent features. Although the pattern of the residuals is not random, especially for components $X$ and $Y$, the particular effect of track offsets is mainly eliminated. This is due to the configuration of the Swarm mission, in particular due to its two satellites, Alpha and Charlie, that fly side by side. Given the large-scale geometry and the longitudinal symmetry of the ring current's magnetic effect at LEO altitude, taking the across-track dif- ferences of Alpha and Charlie measurements is an efficient mean to reduce its effect in lithospheric field models.

The second aspect concerns the flexibility of regional modeling in the use of regularization. As shown in Fig. 6, the regional model carries more energy than the global ones over the bandwidth constrained by near-surface measurements. Given that these are scalar values, the global models WDMAMv2-SH800 and EMM2017 were derived under some regularization constraints (Lesur et al., 2016; Chulliat, 2017) in order to deal with the Backus effect, whose signature is particularly prominent around the Equator (Backus, 1970). Our regional model does not exhibit such artifacts, although we did not apply any regularization. We conclude from the above that regional modeling offers the flexibility of applying regularization that is appropriate to the region of interest instead of a generic regularization that affects the model globally while seeking to smooth out small-scale local artifacts.

The third aspect concerns the estimation of the magnetic thickness and magnetization through spectral analysis of lithospheric field models. Thébault and Vervelidou (2015) noted that the information about the magnetic thickness lies principally in the SH degree at which the peak of the power spectrum occurs. Moreover, this generally falls within the bandwidth known as the spectral gap to denote that it corresponds to spatial scales poorly constrained both by satellites and near-surface measurements. In addition to this uncertainty, independent estimates of the magnetic thickness and magnetization require lithospheric field models of very high resolution. As already noted in Sect. 4.2, our model fully resolves spatial scales up to SH degree 340 . Pushing this limit to higher degrees and/or closing down the spectral gap will eventually modify the shape of the power spectrum and therefore our magnetization and magnetic thickness estimates. Here we found that the $3 \sigma$ confidence interval for the magnetization and magnetic thickness is $[0.6,0.9] \mathrm{A} \mathrm{m}^{-1}$ and $[11,22] \mathrm{km}$, respectively. The estimate of the magnetization is 2 to 3 times larger than the global average as estimated by Thébault and Vervelidou (2015), while the magnetic thickness lies below the global average. Considering the cratonic nature of the southern African crust, this result might reflect an inadequate separation between the magnetization and the magnetic thickness.

Finally, the high-resolution vectorial maps of the magnetic field model displayed at different altitudes offer important insights concerning the sources of the observed anomalies. Although a thorough description and interpretation of these maps is beyond the scope of our study, we point out some striking features of the regional lithospheric field. Some of the prominent anomalies visible in the $X$ component, shown in Fig. 4, include the east-west anomaly along the Damara Belt in Namibia, the Beattie anomaly in South Africa, and the magnetic anomaly along the Limpopo Belt that lies between the Zimbabwe and the Kaapvaal Craton. The first two appear to be relatively shallow features because they are not detected 
above $50 \mathrm{~km}$ of altitude (see Fig. 5). However, the anomaly along the Limpopo Belt persists at higher altitudes, suggesting a deeper and/or elongated origin of the corresponding magnetized structure. The $Y$ component brings forth oceanic magnetic lineations along the west coast of South Africa and Namibia and prominent anomalies along the east borders of the Kaapvaal Craton and into the Mozambique Belt. The anomaly over Mozambique beyond $50 \mathrm{~km}$ of altitude merges with the anomaly over the Limpopo Belt. As expected, all these anomalies are also visible in the $Z$ component. A strong anomaly visible in all three components is a north-south structure in the north-central part of South Africa. We argue that the source of this anomaly is near-surface and smallscale, firstly because this anomaly is clearly seen in the residuals of the WDMAM grid (see Fig. 2d) and secondly because looking at Fig. 5, we observe that it is only visible up to $50 \mathrm{~km}$ of altitude, and then it merges with anomalies of deep origin, possibly related to the Limpopo Belt. Its location coincides with an important band iron formation (see, e.g., Klein and Beukes, 1989).

\section{Conclusions and perspectives}

In this study we jointly modeled satellite, aeromagnetic, marine, and ground magnetic field measurements and derived a lithospheric magnetic field model over southern Africa. We showed that all different data sets are highly compatible with each other, with the horizontal satellite components being the least compatible with the rest of the data set due to external field contamination. We showed that Swarm across-track differences at midlatitudes efficiently eliminate the offsets between adjacent satellite tracks due to the rapid variations of the ring current (see Fig. 2). Mutual exchange of expertise among specialists in internal and external magnetic field sources will help further address the issue of the external field contamination of internal field models (see Stolle et al., 2018, for a relevant compilation of articles).

By means of the R-SCHA power spectrum we showed that our model carries more energy over the bandwidth constrained by near-surface measurements than global highresolution lithospheric field models over the same region, as it does not require regularization (see Fig. 6). The extension of our work to a global model through successive regional analyses is straightforward (see Thébault, 2006, and Thébault et al., 2016, for such studies based on satellite data). The spectral content of such a global model can be evaluated regionally by means of the R-SCHA power spectrum. This is crucial because contrary to satellite data that have an almost homogeneous data coverage, grids based on nearsurface data are extremely inhomogeneous in terms of data quality, availability, and spatial resolution (see Vervelidou and Thébault, 2015, for a regional spectral analysis of the NGDC-720 model).
A wealth of information about the sources of lithospheric field anomalies can be extracted from our regional, highresolution, vector lithospheric field model that can be accurately upward and downward continued (see Figs. 4 and 5). A comprehensive interpretation of these anomalies will benefit from a joint analysis of different geophysical data sets, like gravity, heat flux, and seismic data (see Webb, 2009, for a compilation of such data sets over southern Africa).

Through a spectral analysis of our model we inferred that the mean magnetic thickness and mean magnetization over southern Africa lies between 11 and $22 \mathrm{~km}$ and 0.6 and $0.9 \mathrm{~A} \mathrm{~m}^{-1}$, respectively. More accurate mean estimates can be obtained by means of lithospheric models of higher spatial resolution and more realistic expressions of the power spectrum of the Earth's lithospheric magnetic field. In addition, new spectral tools defined on spherical coordinates will help bridge the gap between Fourier power spectra, which have the ability to represent the energy over small regions but rely on the flat Earth approximation, and the R-SCHA power spectra that provide average estimates over large regions.

Data availability. Grids of our lithospheric field model at various altitudes are available in the Supplement.

\section{The Supplement related to this article is available online at https://doi.org/10.5194/se-9-897-2018-supplement.}

Author contributions. FV and ET designed the study and prepared the necessary code. ET provided the processed Swarm magnetic field data. MK provided the processed surface magnetic field data. FV ran the calculations. FV, ET, and MK analyzed the results. FV wrote the paper with input from ET and MK.

Competing interests. The authors declare that they have no conflict of interest.

Special issue statement. This article is part of the special issue "Dynamics and interaction of processes in the Earth and its space environment: the perspective from low Earth orbiting satellites and beyond". It is not associated with a conference.

Acknowledgements. The authors wish to thank Vincent Lesur for making available the processed CHAMP satellite data set and the South African National Space Agency (SANSA) Space Science in Hermanus for the collaborative effort to obtain and process the southern African repeat station data. Foteini Vervelidou was partly funded by Région Île-de-France and partly by the Deutsche Forschungsgemeinschaft (DFG, German Research Foundation) within the Schwerpunktprogramm 1788 "DynamicEarth" under the 
grant LE2477/7-1. This study was partly funded by CNES in the framework of the Swarm project.

The article processing charges for this open-access

publication were covered by a Research

Centre of the Helmholtz Association.

Edited by: Nicolas Gillet

Reviewed by: Dhananjay Ravat and one anonymous referee

\section{References}

Backus, G., Parker, R., and Constable, C.: Foundations of Geomagnetism, Cambridge University Press, 1996.

Backus, G. E.: Non uniqueness of the external geomagnetic field determined by surface intensity measurements, J. Geophys. Res., 75, 6339-6341, 1970.

Bansal, A. R., Gabriel, G., Dimri, V. P., and Krawczyk, C. M.: Estimation of depth to the bottom of magnetic sources by a modified centroid method for fractal distribution of sources: An application to aeromagnetic data in Germany, Geophysics, 76, L11-L22, 2011.

Barritt, S. D.: African Magnetic Mapping Project (AMMP), ITC journal, 2, 122-131, 1993.

Blakely, R. J.: Potential Theory in Gravity and Magnetic Applications, Cambridge University Press, 1996.

Bouligand, C., Glen, J. M. G., and Blakely, R. J.: Mapping Curie temperature depth in the western United States with a fractal model for crustal magnetization, J. Geophys. Res., 114, B11104, https://doi.org/10.1029/2009JB006494, 2009.

Chulliat, A.: High-resolution crustal field models from the Earth Magnetic Anomaly Grid, IAGA2017 Assembly, Cape Town, A34-1190, 2017.

Dyment, J., Choi, Y., Hamoudi, M., Lesur, V., and Thébault, E.: Global equivalent magnetization of the oceanic lithosphere, Earth Planet. Sc. Lett., 430, 54-65, https://doi.org/10.1016/j.epsl.2015.08.002, 2015.

Farquharson, C. G. and Oldenburg, D. W.: Non-linear inversion using general measures of data misfit and model structure, Geophys. J. Int., 134, 213-227, 1998.

Finlay, C. C., Lesur, V., Thébault, E., Vervelidou, F., Morschhauser, A., and Shore, R.: Challenges handling magnetospheric and ionospheric signals in internal geomagnetic field modelling, Space Sci. Rev., 206, 157-189, https://doi.org/10.1007/s11214016-0285-9, 2017.

Friis-Christensen, E., Lühr, H., and Hulot, G.: Swarm: A constellation to study the Earthś magnetic field, Earth Planets Space, 58, 351-358, 2006.

Haines, G. V.: Spherical Cap Harmonic Analysis, J. Geophys. Res., 90, 2583-2591, 1985.

Hamilton, B.: Rapid modelling of the large-scale magnetospheric field from Swarm satellite data, Earth Planets and Space, 65, 1295-1308, 2013.

Hemant, K. and Maus, S.: Geological modeling of the new CHAMP magnetic anomaly maps using a Geographical Information System (GIS) technique, J. Geophys. Res., 110, B12103, https://doi.org/10.1029/2005JB003837, 2005.
Klein, C. and Beukes, N. J.: Geochemistry and sedimentology of a facies transition from limestone to iron-formation deposition in the early proterozoic Transvaal Supergroup, South Africa, Econ. Geol., 84, 1733-1774, 1989.

Korhonen, J. K., Fairhead, J., Hamoudi, M., Hemant, K., Lesur, V., Mandea, M., Maus, S., Purucker, M. E., Ravat, D., Sazonova, T., and Thébault, E.: Magnetic Anomaly Map of the World-Carte des Anomalies Magnétiques du Monde, scale: 1:50,000,000, 1st edition, 2007.

Korte, M. and Mandea, M.: Geopotential field anomalies and regional tectonic features - two case studies: southern Africa and Germany, Solid Earth, 7, 751-768, https://doi.org/10.5194/se-7751-2016, 2016.

Korte, M., Mandea, M., Kotzé, P., Nahayo, E., and Pretorius, B.: Improved observations at the southern African geomagnetic repeat station network, S. Afr. J. Geol., 110, 175-186, 2007.

Kother, L.: Regional lithospheric field modelling using monopoles and a combination of airborne and satellite data, IAGA2017 Assembly, Cape Town, A34-603, 2017.

Kother, L., Hammer, M. D., Finlay, C. C., and Olsen, N.: An equivalent source method for modelling the global lithospheric magnetic field, Geophys. J. Int., 203, 553-566, 2015.

Kotsiaros, S. and Olsen, N.: The geomagnetic field gradient tensor, GEM-International Journal on Geomathematics, 3, 297-314, 2012.

Kunagu, P., Balasis, G., Lesur, V., Chandrasekhar, E., and Papadimitriou, C.: Wavelet characterization of external magnetic sources as observed by CHAMP satellite: evidence for unmodelled signals in geomagnetic field models, Geophys. J. Int., 192, 946-950, 2013.

Laundal, K. M. and Richmond, A. D.: Magnetic coordinate systems, Space Sci. Rev., 206, 27-59, https://doi.org/10.1007/s11214016-0275-y, 2017.

Lesur, V., Wardinski, I., Rother, M., and Mandea, M.: GRIMM The GFZ Reference Internal Magnetic Model based on vector satellite and observatory data, Geophys. J. Int., 173, 382-394, 2008.

Lesur, V., Wardinski, I., Hamoudi, M., and Rother, M.: The second generation of the GFZ Reference Internal Magnetic Model: GRIMM-2, Earth, Planets and Space, 62, 765-773, 2010.

Lesur, V., Rother, M., Vervelidou, F., Hamoudi, M., and Thébault, E.: Post-processing scheme for modelling the lithospheric magnetic field, Solid Earth, 4, 105-118, https://doi.org/10.5194/se-4105-2013, 2013.

Lesur, V., Hamoudi, M., Choi, Y., Dyment, J., and Thébault, E.: Building the second version of the World Digital Magnetic Anomaly Map (WDMAM), Earth, Planets and Space, 68, https://doi.org/10.1186/s40623-016-0404-6, 2016.

Lowes, F. J.: Mean-square values on sphere of spherical harmonic vector fields, J. Geophys. Res., 71, 2179, https://doi.org/10.1029/JZ071i008p02179, 1966.

Mandea, M., Panet, I., Lesur, V., De Viron, O., Diament, M., and Le Mouël, J. L.: Recent changes of the Earth's core derived from satellite observations of magnetic and gravity fields, P. Natl. Acad. Sci., 109, 19129-19133, 2012.

Maus, S.: An ellipsoidal harmonic representation of Earth's lithospheric magnetic field to degree and order 720, Geochem. Geophy. Geosy., 11, https://doi.org/10.1029/2010GC003026, 2010. 
Maus, S., Gordon, D., and Fairhead, D.: Curie-temperature depth estimation using a self-similar magnetization model, Geophys. J. Int., 129, 163-168, 1997.

Maus, S., Lühr, H., Rother, M., Hemant, K., Balasis, G., Ritter, P., and Stolle, C.: Fifth-generation lithospheric magnetic field model from CHAMP satellite measurements, Geochem. Geophy. Geosy., 8, https://doi.org/10.1029/2006GC001521, 2007.

Maus, S., Yin, F., Lühr, H., Manoj, C., Rother, M., Rauberg, J., Michaelis, I., Stolle, C., and Müller, R. D.: Resolution of direction of oceanic magnetic lineations by the sixthgeneration lithospheric magnetic field model from CHAMP satellite magnetic measurements, Geochem. Geophy. Geosy., 9, https://doi.org/10.1029/2008GC001949, 2008.

Maus, S., Barckhausen, U., Berkenbosch, H., Bournas, N., Brozena, J., V., C., Dostaler, F., Fairhead, J. D., Finn, C., Von Frese, R. R. B., Gaina, C., Golynsky, S., Kucks, R., Lühr, H., Milligan, P., Mogren, S., Müller, R. D., Olesen, O., Pilkington, M., Saltus, R., Schreckenberger, B., Thébault, E., and Caratori Tontini, F.: A 2-arc min resolution Earth Magnetic Anomaly Grid compiled from satellite, airborne, and marine magnetic measurements, Geochem. Geophy. Geosy., 10, https://doi.org/10.1029/2009GC002471, 2009.

Olsen, N., Friis-Christensen, E., Floberghagen, R., Alken, P., Beggan, C., Chulliat, A., Doornbos, E., Da Encarnação, J., Hamilton, B., Hulot, G., and Van den Ijssel, J., Kuvshinov, A., Lesur, V., Lühr, H., Macmillan, S., Maus, S., Noja, M., Olsen, P. E. H., Park, J., Plank, G., Püthe, C., Rauberg, J., Ritter, P., Rother, M., Sabaka, T. J., Schachtschneider, R., Sirol, O., Stolle, C., E. Thébault, Thomson, A. W. P., Tøffner-Clausen, L., Velímský, J., Vigneron, P., and Visser, P. N.: The Swarm satellite constellation application and research facility (SCARF) and Swarm data products, Earth Planets and Space, 65, 1189-1200, 2013.

Olsen, N., Ravat, D., Finlay, C. C., and Kother, L. K.: LCS-1: a highresolution global model of the lithospheric magnetic field derived from CHAMP and Swarm satellite observations, Geophys. J. Int., 211, 1461-1477, 2017.

Plattner, A. and Simons, F. J.: Spatiospectral concentration of vector fields on a sphere, Applied and Computational Harmonic Analysis, 36, 1-22, https://doi.org/10.1016/j.acha.2012.12.001, 2014.

Plattner, A. and Simons, F. J.: Internal and external potentialfield estimation from regional vector data at varying satellite altitude, Geophys. J. Int., 211, 207-238, https://doi.org/10.1093/gji/ggx244, 2017.

Rajaram, M., Anand, S. P., Hemant, K., and Purucker, M. E.: Curie isotherm map of Indian subcontinent from satellite and aeromagnetic data, Earth Planet. Sc. Lett., 281, 147-158, 2009.

Ravat, D., Whaler, K. A., Pilkington, M., Sabaka, T., and Purucker, M.: Compatibility of high-altitude aeromagnetic and satellitealtitude magnetic anomalies over Canada, Geophysics, 67, 546554, 2002.

Ravat, D., Pignatelli, A., Nicolosi, I., and Chiappini, M.: A study of spectral methods of estimating the depth to the bottom of magnetic sources from near-surface magnetic anomaly data, Geophys. J. Int., 169, 421-434, 2007.

Reigber, C., Lühr, H., and Schwintzer, P.: CHAMP mission status, Adv. Space Res., 30, 129-134, 2002.

Rother, M., Lesur, V., and Schachtschneider, R.: An algorithm for deriving core magnetic field models from the Swarm data set, Earth, Planets and Space, 65, 1223-1231, 2013.
Sabaka, T. J., Olsen, N., and Purucker, M. E.: Extending comprehensive models of the Earth's magnetic field with Øersted and CHAMP data, Geophys. J. Int., 159, 521-547, 2004.

Sabaka, T. J., Olsen, N., Tyler, R. H., and Kuvshinov, A.: CM5, a pre-Swarm comprehensive geomagnetic field model derived from over $12 \mathrm{yr}$ of CHAMP, Ørsted, SAC-C and observatory data, Geophys. J. Int., 200, 1596-1626, 2015.

Salem, A., Green, C., Ravat, D., Singh, K. H., East, P., Fairhead, J. D., Mogren, S., and Biegert, E.: Depth to Curie temperature across the central Red Sea from magnetic data using the defractal method, Tectonophysics, 624, 75-86, 2014.

Scheiber-Enslin, S., Ebbing, J., and Webb, S. J.: An integrated geophysical study of the Beattie magnetic anomaly, South Africa, Tectonophysics, 636, 228-243, 2014.

Schott, J. J. and Thébault, E.: Geomagnetic Observations and Models, IAGA, Special Sopron Book Series, vol. 5, chap. Modelling the earth's magnetic field from global to regional scales, 229264, Springer, 2011.

Stettler, E. H., Fourie, C. J. S., and Cole, P.: Total magnetic field intensity map of the Republic of South Africa (in 4 panels), Council for Geoscience, Pretoria, 2000.

Stolle, C., Olsen, N., Richmond, A., and Opgenoorth, H. (Eds.): Earth's Magnetic Field: Understanding Geomagnetic Sources from the Earth's Interior and Its Environment, Springer, 2018.

Thébault, E.: Global lithospheric magnetic field modelling by successive regional analysis, Earth Planets and Space, 58, 485-495, 2006.

Thébault, E. and Vervelidou, F.: A statistical spatial power spectrum of the Earth's lithospheric magnetic field, Geophys. J. Int., 201, 605-620, 2015.

Thébault, E., Schott, J. J., and Mandea, M.: Revised spherical cap harmonic analysis (R-SCHA): Validation and properties, J. Geophys. Res., 111, B01102, https://doi.org/10.1029/2005JB003836, 2006.

Thébault, E., Vervelidou, F., Lesur, V., and Hamoudi, M.: The along-track satellite analysis in planetary magnetism, Geophys. J. Int., 188, 891-907, 2012.

Thébault, E., Vigneron, P., Langlais, B., and Hulot, G.: A Swarm lithospheric magnetic field model to SH degree 80, Earth Planets and Space, 68, https://doi.org/10.1186/s40623-016-0510-5, 2016.

Thébault, E., Lesur, V., Kauristie, K., and Shore, R.: Magnetic Field Data Correction in Space for Modelling the Lithospheric Magnetic Field, Space Sci. Rev., 206, 191-223, https://doi.org/10.1007/s11214-016-0309-5, 2017.

Thomson, A. W. P. and Lesur, V.: An improved geomagnetic data selection algorithm for global geomagnetic field modelling, Geophys. J. Int., 169, 951-963, 2007.

Vervelidou, F.: Contribution à la modélisation et à l'interprétation multi-échelle du champ magnétique de la lithosphère terrestre, Ph.D. thesis, Université Paris Diderot - Paris 7, 2013.

Vervelidou, F. and Thébault, E.: Global maps of the magnetic thickness and magnetization of the Earth's lithosphere, Earth Planets and Space, 67, https://doi.org/10.1186/s40623-015-0329-5, 2015.

Voorhies, C. V., Sabaka, T. J., and Purucker, M.: On magnetic spectra of Earth and Mars, J. Geophys. Res.-Planets, 107, https://doi.org/10.1029/2001JE001534, 2002. 
Webb, S. J.: The use of potential field and seismological data to analyze the structure of the lithosphere beneath southern Africa, Ph.D. thesis, University of the Witwatersrand, 2009.

Whaler, K. A.: Downward continuation of Magsat lithospheric anomalies to the Earth's surface, Geophys. J. Int., 116, 267-278, 1994.
Whaler, K. A.: CHAMP Mission Results I, chap. Crustal magnetization distribution deduced from CHAMP data, 281-287, Springer, New York, 2003. 